Check for updates

Cite this: J. Mater. Chem. B, 2020, 8, 7143

Received 14th June 2020,

Accepted 6th July 2020

DOI: 10.1039/d0tb01491e

rsc.li/materials-b

\section{Specifiable biomimetic microsponges for timed release of crystal entrapped biomolecules useful in bone repair}

\author{
David W. Green, (D) *a Artemis Stamboulis $\left(\mathbb{D}^{\mathrm{a}}{ }^{\mathrm{a}}\right.$ and Besim Ben-Nissan (D) ${ }^{\mathrm{b}}$
}

\begin{abstract}
Most marine materials, by nature, contain crystals of inorganic matter with specific structures that allow the loading, release, and delivery of biomolecules that can be utilized in clinical applications. These structures can be biomimetically synthesized. Aggregates of inorganic particles generated by biomimetic microsponges may provide surfaces and structures for cell attachment, organization, and promotion of matrix synthesis. Biomimetic microsponges have been developed with tunable release profiles differing by the rate (speed over distance), velocity (rate of change in direction), and the quantity discharged over time, according to biomolecular species. Specifically, the types of proteins involved guide and regulate cells in physical contact with the microsponges, for instance, reprogramming somatic cells, the switching phenotypes, or specifying stem cell differentiation. Applications for these microsponges include gene transfection of localized cells and promotion of bone matrix synthesis by the externalized display of RGD cell adhesive peptides and the release of crystal entrapped, occluded, adsorbed and infused rhBMP-2 and plasmid. A requirement for de novo bone formation is a solid structure to enable osteocytes to lay new bone tissue. In this study, biomimetic microsponges highlight tremendous potential as osteoconductive packing material in bone repair with parallel influence on regeneration. Majorly, microsponges offer pronounced osteoinductivity, unlike many other bone particulates, by solid-state integration of active regenerative biological molecules through the prism of the biomineral crystalline structure.
\end{abstract}

\section{Introduction}

The ability to generate new bone and bone substitutes for skeletal repair, replacement, and restoration is a tremendous

\footnotetext{
${ }^{a}$ Biomaterials Group, School of Metallurgy and Materials, University of Birmingham. Edgbaston, Birmingham B15 2SE, UK.

E-mail: d.w.green.1@bham.ac.uk

${ }^{b}$ University of Technology, Sydney, School of Life Sciences, Translational Biomaterials and Medicine Group, Broadway, 2007 NSW, Australia

$\dagger$ Electronic supplementary information (ESI) available. See DOI: 10.1039/ dotb01491e
}

clinical need of high importance. ${ }^{1,2}$ Conventional allograft substitutes frequently do not bridge and unify with host bone, which might cause new infections and continue to create fractures. ${ }^{3}$ The relative paucity of techniques to repair musculoskeletal tissues in this manner necessitates new, inventive, and more effective clinically acceptable approaches. ${ }^{3,4}$ Many product solutions have not shown sufficient evidence of effectiveness to warrant clinical procedures. ${ }^{5}$ The lack of availability and supply of appropriate allograft generated the need for the production of synthetic materials. The synthetic particulate and solid bone void fillers promise affordable, specifiable bone replacements without biological complications. ${ }^{6}$ To fully deliver the overall and sound new bone regeneration, the tissue engineering scaffolds must possess appropriate interaction and bioactivity in the regeneration of surrounding bone cells and tissues. There merely exist a few examples of particulate bone substitutes possessing cell bioactivities, impregnated with therapeutic drugs such as antibiotics into a range of composites made of calcium phosphates, including carbonated apatite ${ }^{7}$ and proteins which lead to translocation, rather than the mineral ions that only stimulate bone regeneration. A fine example of the new generation materials include composites containing bioactive calcium phosphates, bioglasses, nanobioceramics, and calcium silicate concentric spheres. ${ }^{8,9}$ A principal way that these materials achieve this is by integrating and infusing bioactive molecules (from small 13 kDa morphogens, 283-396 amino acids, enzymes $13.7 \mathrm{kDa}$ to hemoglobin $64.5 \mathrm{kDa}$ at $5 \mathrm{~nm}$ diameter) to deep into the material itself so that release occurs regularly, with first-order kinetics (hence time-dependent), in the long term by slow crystal disaggregation, dissolution, and resorption in a physiological setting.

In our previous work, porous calcium carbonate (vaterite is an unstable $\mathrm{CaCO}_{3}$ polymorph) microsponges, with biomimetic morphologies (in terms of shape, size, pore arrangementsemulating fossilized shells of foraminiferan (O. universa $\mathrm{CaCO}_{3}$ exoskeletons and Coccolithophores) have been shown to promote in vitro, the early phases of human bone tissue formation. ${ }^{7,10}$ As a natural corollary to this study, nacre and a majority of other 
biomineralized structures entrap proteins between their crystal plates and infuse intracrystalline proteins because of their crystal controlling faculties. ${ }^{11-13}$ Nacre has been reported to release signaling proteins (three gigasin-2 isoforms and a cystatin A2 in C. gigas) in bone capable of inducing new bone formation and remodeling. ${ }^{14}$

In this study the utility and functionality of marine shells of fossilized foraminiferan like vaterite microsponges (Fig. 1 and $2 \mathrm{~A} v s$. B) have been extended from a simple osteoconductive cell framework, albeit loosely held together, to provide a very efficient and most importantly an osteoinductive lattice framework toward a clinically more appropriate potential tissueengineered bone void filler. The structure is further functionalized for cell boosting interactions by controlled release of occluded and entrapped morphogens, DNA, and an adhesive peptide (Fig. 1). These structural inorganic materials can have an additional capacity to transiently transfect cells embedded in polysaccharide (specifically alginate and chitosan) hydrogel in vivo. This same entrapment mechanism, highlighted in Fig. 1, is usable for tethering bio-active molecules at the exterior surface of the microsponge to increase cell interactions. A right candidate is the cell adhesive tripeptide peptide, arginylglycylaspartic acid complex $\left(\mathrm{C}_{12} \mathrm{H}_{22} \mathrm{~N}_{6} \mathrm{O}_{6}\right.$; RGD) (Fig. 1C).

Our biomimetically produced space filler having osteoinductive effects can be designed with more options through a tightening of the parameters for chemical morphosynthesis, component sizes, and higher-scale microsponge morphology. The parametric tuning of chemical morphosynthesis translates to the custom programming for how specific entrapped proteins are released over time in zero order or first order kinetics.

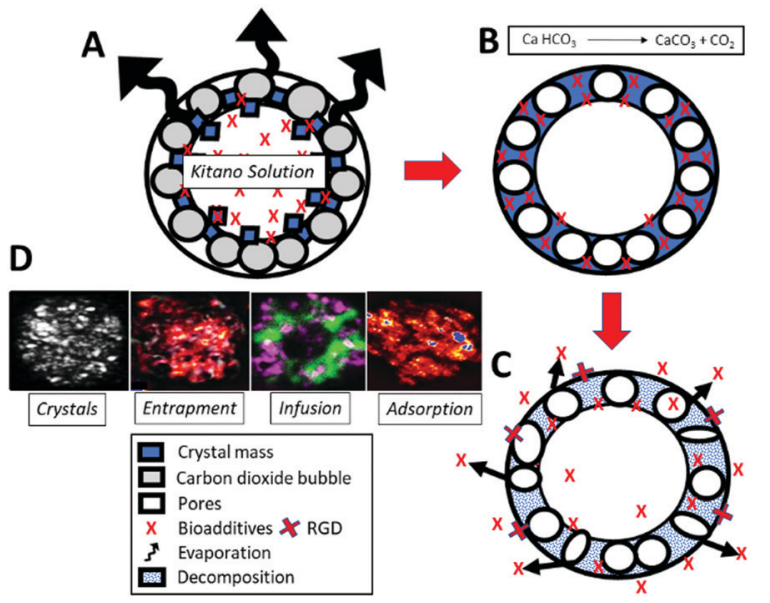

Fig. 1 Scheme of vaterite microsponge formation chemistry by Walsh et al. (A) Evaporation of enclosed Kitano droplets from prepared reverse micelles drives crystallisation in (B) and the entrapment (ii), infusion and adsorption (iv) of Kitano mixed encapsulates. ${ }^{15}$ Schematic part $\mathrm{C}$ describes the swift dissolution of the unstable vaterite mineral leading to the destruction of the sponge and corresponding release of the previously entrapped biomolecules into the external environment. The exterior texture, porosity, chemical make-up and display of adhesive peptides facilitate cell adhesion and growth between them. Finally, in (D) functional highlights of the microsponge crystal structure, and biomolecule entrapment, infusion and adsorption. ${ }^{16}$

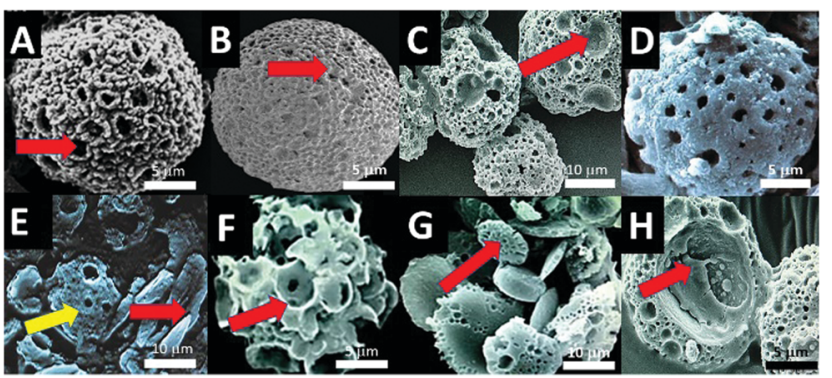

Fig. 2 Improvements to microsponges synthesis; (A) enlarged porous microsponges from standard $10 \mu \mathrm{m}$ diameter sponges to $20 \mu \mathrm{m}$ sponges; (B) large microsponge with entirely new architecture analogous to a species of fossilized foraminiferans (red arrow pointing to a large micropore); (C), group of enlarged microsponges showing the extra variation in pore structure consisting of medium and very large surface pores (red arrow pointing to a large micropore); (D), degrading microsponge at 7 days in culture media; (E) degradation of microsponges (yellow arrow) into calcite shards (red arrow); (F) microporous spheres generated in corn oil (red arrow pointing to a large pore); (G) porous and partially porous microdiscs (red arrow pointing at representative microdisc with submicron pores); (H) opened-out microsponges containing (entrapped) protein indicated by a red arrow.

Osteoinductivity is provided by entrapment, inclusion and occlusion, as well as infusion and adsorption (Fig. 1) of critical regenerator drugs, genes, growth factors and other proteins active in bone cell control and tissue formation. One major candidate for bone repair is bone morphogenic protein (BMP).

\section{Experimental work}

In this study, we revised the inceptive method for microsponge synthesis by Walsh et al. ${ }^{15}$ (Fig. 1A-C) to increase microsponge size over the previously attained maximum of $10 \mu \mathrm{m}$, and generating new biomolecular capturing, releasing, and osteoinductive, biomimetic morphologies. Microsponge size, pore structure, and shape were modulated (Fig. 2A vs. C) by adjusting drying rates (from a standard $25^{\circ} \mathrm{C}$ graduated to $37^{\circ} \mathrm{C}$ ) and heating times (from standard $24 \mathrm{~h}$ to $72 \mathrm{~h}$ ) and by increasing the quantity of alkane hydrocarbon oils by $5 \%$ in the reverse microemulsion. New drying and heating levels did not significantly reduce the microsponge total yield, which consistently averaged between $1 \mathrm{~g}$ to 2 g-equating to a $5 \mathrm{~mm}$ diameter pellet from the original $50 \mathrm{~mL}$ water-in-oil emulsion. Larger microsponges ranging between 5-30 $\mu \mathrm{m}$ (Fig. 2A-C) were developed from microemulsions with slightly elevated (by $5 \%$ volume) quantities of dodecane $\left(\mathrm{C}_{12} \mathrm{H}_{26}\right)$, and octadecane $\left(\mathrm{C}_{18} \mathrm{H}_{38}\right)$. One fundamental rationale of a biomimetic approach to materials chemistry is to limit the use of synthetic and toxic chemicals and employ environmentally benign reactants in green chemistry.

As a proof-of-concept, microemulsions emerged from: (a) sodium lauryl sulphate (SDS)/aqueous Kitano based micelles immersed in vegetable (corn, Zea mays; C16:0 [80\%]/C18:0 $[14 \%] / \mathrm{C} 20: 0[3 \%] / \mathrm{C} 18: 1$ cis-9/C18:2 $n-6)$ oil and (b) phospholipid source (from soybean, Glycine $\max ; \mathrm{C}_{57} \mathrm{H}_{98} \mathrm{O}_{12} ; \mathrm{C}-18: 3[7 \%] /$ C-18:2 [51\%]/C-18:1 [23\%]/C-18:0 [4\%]/C-16:0 [10\%]) were 
produced in standard micellar Kitano droplets in an alkane hydrocarbon oil (Fig. 1F). ${ }^{15,17}$

Using the two separate mixtures porous spheroids were generated with strikingly different architectures and porosity levels, pore packing distributions, and crystal sizes. Although the average yield of microsponges was analogous, there was a greater variety of surface and volume morphologies-varying between spheres, discs and shards (Fig. 2A versus B-D).

\section{Results and discussions}

Microsponges proved to be efficient at slow and controlled release of several proteins. The generated architecture of microsponge struts, pores and channels further organised and elaborated itself by the inclusion of several biological proteins that modified crystal growth and shape. Hence determining the type of crystal morphotype and the time that it is made during microsponge morphogenesis where along the boundary of the surfactant template it is deposited and how dense the crystal packing. Even the non-crystallisation associated therapeutic protein BMP, DNA and RGD tripeptide modulated the inorganic crystallization leading to structural changes to the microsponge architecture.

Fig. 2G shows the structure of the shell of nacre Pinctada fucata, its water-soluble matrix (WSM), which is a complex combination of glycoproteins ${ }^{18,19}$ and chitin $\left.\left(\mathrm{C}_{8} \mathrm{H}_{13} \mathrm{O}_{5} \mathrm{~N}\right)_{n}\right)$. Micelles containing water-soluble matrix extracted from this nacre $\left(2 \mathrm{mg} \mathrm{mL} \mathrm{mL}^{-1}\right.$ total protein) gave rise to nodules of plated structures and spheres of intercalated rounded plates (Fig. 2G). When chitin nanofibrils, extracted by ultrasonication in mild $\mathrm{pH}$ four acid (30-800 $\mathrm{nm} ; 20 \mathrm{~mL}$ per $\mathrm{mL}$ aqueous solvent) and adding it into the mixture with WSM, a fascinating nacremimicking multi-laminated calcium carbonate block assembly were synthesized, highlighting the strong influence on crystal growth aspects and habit formation (Fig. 2E-G). The vaterite microsponges produced shown in Fig. 2D and E, however, were more stable than calcite shards and blocks observed in (Fig. 2H). Also, it was seen that other proteins and short sequenced peptides such as RGD tripeptides $\left(<0.3 \mathrm{mg} \mathrm{mL}{ }^{-1}\right)$ could entrap themselves into the crystal structure and absorb into the internal surfaces (Fig. $2 \mathrm{H}$ ).

To observe the morphological changes, biomolecules with a broad range of sizes, encompassing many essential functional proteins and nucleic acid polymers (26-1000 kDa), were integrated deep into the microsponge structure (Fig. 2H). Our calculations came up with a $25 \%$ capture efficiency for DNA plasmids incorporated one-by-one into a population of three million microsponges. There is a tremendous variance in capture rates between biomolecules of different morphologies, sizes, and folding patterns. So, for example, RNAse enzyme was captured with a 55\% efficiency (Fig. 2H). Analogously, natural diatom micro skeletons with comparable size and porosity possess capture efficiency of $14-15 \%$.

Essential molecules for bioengineering with cells for tissue reconstruction centre on informational DNA and instructional morphogens. DNA or rhBMP-2 containing microsponges, release the constituents in parallel with swift decomposition of metastable vaterite. In a tissue engineering context triggered decomposition and rupturing is important for cell and tissue control and guidance. Previously, we have occluded paramagnetic particles into the microsponge structure and shown immediate damage when put through a strong magnetic field. ${ }^{5}$ There is more biomimetic acting advantage for release propelled and guided by resorption and chemical reactions. ${ }^{11}$

\section{Loading of non-porous $v s$. porous microsponges}

The release of a non-protein $p$-nitrophenyl phosphate disodium salt (PNPP; $\mathrm{C}_{6} \mathrm{H}_{6} \mathrm{NO}_{6} \mathrm{P} ; 371 \mathrm{MW}$ ) incorporated into both porous and solid (non-porous) microsponge formation was detected at $405 \mathrm{~nm}$ and tracked, over four days towards the $100 \%$ release mark. Lit. was observed that there was a little difference in the elution profiles solid, to non-porous microsponges compared. This indicates that the PNPP dye was almost exclusively adsorbed onto the crystals and not entrapped (Fig. 3A).

In a further study, a marine structure, non-porous and porous structures (open porous marine sponge (S. officinalis), the non-porous solid vaterite sponges) were compared. After loading of $50 \%$ of the DNA plasmid (combined with LacZ operon), the microsponges were released into PBS solution. In contrast, the porous microsponges showed steady slowrelease on day 8 with a smooth. Shallow sloping profile, on the other hand, the solid, non-porous vaterite spheres released $100 \%$ of the full content in 5 days, showing that it was fully released from its surface (Fig. 3B-D). If we compare this to a useful, very open tissue engineering natural framework
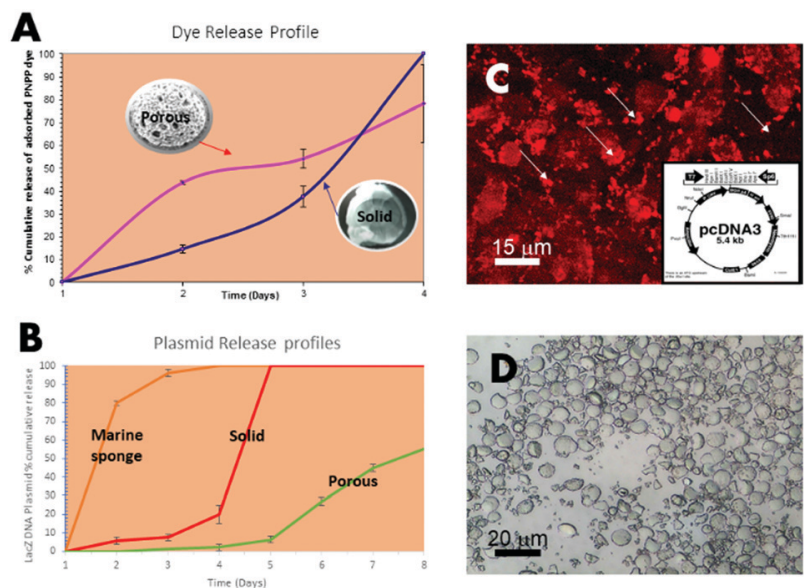

Fig. 3 Release profiles of pore/channel entrapped and adsorbed biomolecules from microsponges as a percentage of the total quantity added at synthesis (pink = microsponges) in parallel with an open porous framework (blue line = solid vaterite spheres); (A) adsorbed PNPP dye release from porous microsponges versus non-porous microsponges; (B) release profile of entrapped LacZ expression vectors/the DNA plasmid construct from an open porous marine sponge (S. officinalis), the non-porous solid vaterite sponges and porous microsponges; (C) DNA plasmid stained with ethidium homodimer-1 showing entrapped and plasmids released into surrounding media; (D) light image of non-porous microsponges as the control. 
designed for cell infiltration, it was noted that $80 \%$ of the content was released within the first two days in a similar PBS solution (Fig. 3B). As observed in this experiment, a fully interconnected porous microsponge structure is a more efficient drug delivery carrier that can allow much slower drug and biomolecule delivery than the solid microsponges in clinical applications.

\section{Gene delivery}

Another approach in drug and gene delivery is to embed the matter to be delivered in a soft hydrogel matrix, which facilitates its transfer to the target. LacZ plasmid (pcDNA3-myc-hislacZ) containing microsponges co-cultured with Chinese hamster ovary cells (CHO) (Fig. 4A-D) were positively transfected, although transiently, in an ex vivo chick defect model and implanted inside a nude-mice (Fig. 4A and B). The transfections were imaged under scanning confocal laser microscopy using a FITC- $\beta$-galactosidase conjugated antibody against the present galactosidase enzymes (Fig. 4C and D) and by GFP expression vectors pcDEST47-GFP. The LacZ $\beta$-galactosidase plasmid crystal entrapment and release to neighbouring cells was demonstrated in vitro, as shown in Fig. 4C. Transfection occurs through the backing of the sodium alginate and chitosan matrices (Fig. 4B red arrows) bearing calcium and chitosan transfection reagents-embedding the microsponge populations at high density (Fig. 4B inside red circle). This simultaneously buffers and translocates the microsponge dispersed plasmid DNA into the cell, further assisted by calcium ions and chitosan in the matrix, leading to a transfection efficiency of $62 \%$, calculated by dividing the numbers between GFP positive and

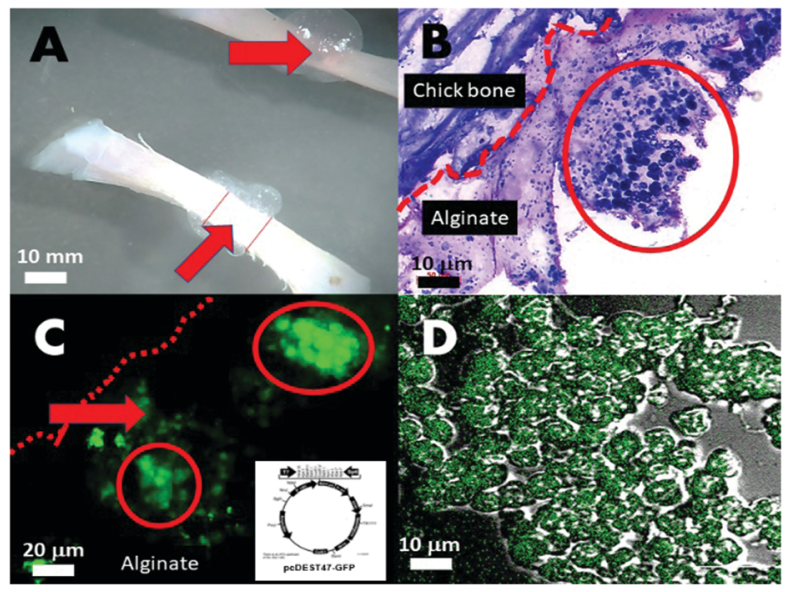

Fig. 4 (A) Microscope view of the whole chickbone and the small defect filled with the polysaccharide carrier matrix embedding the plasmid microsponges; (B) haemotoxylin and eosin stained thinly sliced section through one edge of the defect area showing the (alginate/chitosan) polysaccharide matrix (light pink) embedding the microsponges; (C) fabricated green fluorescence protein expressed by $\mathrm{CHO}$ cells at the defect site indicative of transfection by GFP plasmid (construct design in insert) originally from microsponges; (D) high power confocal fluorescence overlaid with differential interference contrast (DIC) view of clearly delineated $\mathrm{CHO}$ cells expressing green fluorescent protein.
GFP negative cells in 20 random areas of the array at the defect zone viewed through the confocal laser scanning microscope.

Genetic matter and its expression mechanism are sensitive to physical damage. Hence, incorporation into a solid delivery framework by external matter for drug or gene delivery necessarily damages genetic material and disrupts the coded information. Safer methods of fast and efficient gene delivery are in pressing need.

\section{Developing extracellular matrix}

The initiation of mineral nodules from developing extracellular matrix was demonstrated at two weeks in unadulterated microsponges (Fig. 5A-C), RGD (Fig. 5D-F) and rhBMP-2 (Fig. 5G-I) pellets by the presence of dense organized collagen matrix highlighted by intense Sirius red - (for human collagens) and strong birefringence under polarised light (Fig. 5A, B, D, E, G and $\mathrm{H}$ ) and as well as positive, intense patchy staining for collagen I (Fig. 5H). Experimental conditions included the standard operable hBMSC and microsponge centrifuged pellet, made-up of 500 000-700 000 microsponges, and 2 to 2.5 million hBMSC. Individual microsponges were self-supporting and measured $5 \mu \mathrm{m}$ in diameter and centrifuged at $2000 \mathrm{rpm}$. Besides, collagen surface area coverage per single pellet peaked at $80 \%$ on average. In contrast, pellets without microsponges did not synthesize the collagen matrix in such large quantities at 21 days. Only after 21 days did collagen synthesis occur. The presence of collagen was picked-up through specific colorimetric matrix staining of microsponge and hBMSC aggregated pellets for nuclei, cartilage, and bone matrix (Fig. 5C, F and I).

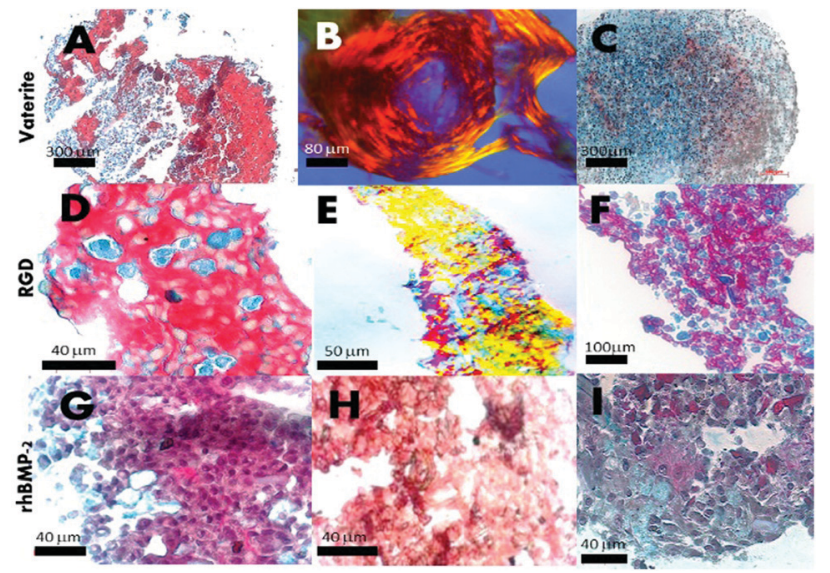

Fig. 5 Three week old standard pellet culture of microsponges entrapping and adsorbing BMP-2 growth factor and RGD with surface display; (A) in vitro bone matrix formation (Sirius red) covering the pellet of human bone marrow stromal cells combined with standard vaterite microsponges; (B) polarised light image of an individual hBMSC/microsponge pellet showing highly ordered collagen matrix; (C) histological image of an individual hBMSC pellet without vaterite microsponge content; (D) bone matrix formation within hBMSC/microsponges containing RGD display; (E) under polarised light conditions; (F) a pellet without RGD display; (G) 3-dimensional hBMSC/microsponge pellet culture with microsponge entrapped rhBMP-2; $(\mathrm{H})$ immunolabelling for bone collagen type 1; (I) histological sections of hBMSC pellet at 21 days stained for Sirius red/alcian blue (A, D, G and C, F, I); (450-750 000 microsponges per pellet). 
Significant collagen formation routinely starts at 28 days in these cultures that are suspended in primary media without osteogenic supplementation. In comparison, hBMSC pellets in osteogenic media $\left(50 \mu \mathrm{g} \mathrm{mL}^{-1}\right.$ ascorbic acid and $10 \mathrm{nM}$ dexamethasone) with and without microsponges showed extensive collagen synthesis highly structured arrangement of cell and fibrillar matrix observed in pellets containing the microsponges (Fig. 5F). At 21 days, 35\% to $45 \%$ of the pellet's surface area comprised dense collagen with localized mineralization traced around degraded microsponges as determined by positive von Kossa staining (data are not shown). The development of structure and cellularity enhances itself in pellets containing microsponges with entrapped rhBMP-2 (Fig. 5G-I). The resultant histological evidence also suggests that a lower microsponge loading enhances collagen synthesis. In the experiment, inside the pellets containing fewer microsponges (approximately $350000)$ as opposed to those. Confined pellets containing 550000 microsponges, the density of collagen matrix synthesis was high probably due to additional restrictions on nutrient exchanges in dense microsponge aggregates. We have also observed compact collagen type I synthesis and mineral precipitation in pellets comprising human bone marrow stromal cells grown in primary media (Fig. 5H red arrows showing denser regions). It can be stated that microsponges provide a focus for and inertia that promotes differentiation of precommitted stromal cells to organize and synthesize a new bone matrix (Fig. 5A-H). Structural cues and the increased concentration of calcium ions are likely to play a role, as explained in Green et al. 2008. ${ }^{5}$

Tissue engineering microsponges can be arranged in cellcontaining hydrogels to boost cell activity. Microsponges have been tested for their capacity to release regenerative proteins inside polysaccharide hydrogels of chitosan and alginate. Hydrogels of this sort provided interactive and cooperative self-contained microenvironments for progenitor cell populations synthesis and assembly. Further to this, microsponge populations precoated with rhBMP-2, also trapped inside the microsponge meso- and nanopores, promoted mineralized bone matrix tissue inside hBMSC containing mineral coated polysaccharide beads [data not shown]. In contrast, polysaccharide beads containing only hBMSC resulted in an organized collagen matrix that was auto-synthesized without mineralization. Microsponges, therefore, have other auxiliary roles partnered with different tissue engineering scaffolds.

Orthopaedic and maxillofacial surgeons, during surgery, introduce bone marrow to the implant-tissue interface for better osteointegration. However, only a few developed or commercially available bone void particulate graft material that needed in the surgery possesses the required integrated biochemical factors that can induce osteoinduction during bone formation. We have demonstrated the simple, albeit in a few ways, the capacity to modulate the release characteristics and propagation profiles from these microsponge structures directly and locally. Shape, size, and architecture changes can influence degradation rates and therefore release of entrapped species. Tests of this capacity focused on a cell adhesion protein, one key morphogen, and DNA fragments, each individual involved in a pathway for cellular change and control of genetic, membrane junction, and membrane receptor pathways. Double-stranded DNA delivery in the form of recombinant plasmid from the microsponges was demonstrated both in vitro solution and then in vivo tissues causing expression of the foreign GFP and LacZ genes. Significantly, in terms of the original mechanics involved in this spontaneous non-viral transient transfection of Chinese hamster ovary cells, which occurred in vivo in the presence of DNA microsponges embedded in a $2 \%$ sodium alginate and chitosan matrix facilitation matrix with calcium and phosphate supplementations. It was also observed that the alginate imparted calcium ions and the chitosan un-crosslinked fragments that bound and translocated DNA into the cells. Surrounding hBMSC and microsponges co-cultures promoted bone matrix synthesis in 1 week ahead of hBMSC pellets. A similar scale of bone matrix synthesis typically occurs between 21-28 days at the earliest.

Although we had no absolute proof that this response is osteoinductive or osteoconductive other than the direct comparison with original microsponges, BMP-2 microsponges show osteoinductive bone formation, with similar histological structures as the RGD microsponges. A requirement for de novo bone formation is a solid structure to enable osteocytes to lay new bone tissue. Aggregates of inorganic particles within microsponges may provide the required structure and surfaces for cell attachment, organization, and promotion of matrix synthesis.

\section{Conclusion}

In conclusion, we have expanded the morphology characters and extended the functional repertoire of microsponges from their original purpose as a packing material to support tissue assembly by physical means. Microsponges made by more benign, green chemical synthesis methods, and greater control of size and morphology, and delivering gene components for transient transfection produced more controlled release of the biomolecules incorporated. Original measurements of controlled release profiles are in first-order kinetics. Tightly packed 3D hBMSC and microsponge pellets appear in the histological examination to promote proper bone matrix synthesis and more extensively when entrapped rhBMP-2 releases itself from neighbouring microsponges. The phenomenon is equally pronounced in microsponges displaying the RGD cell adhesion peptide, which is supposed to increase cell-matrix and cell-cell binding and interactions. The matrix present in the RGD display is more intense and densified than the rhBMP-2 microsponges. It suggests contact and adhesion are more critical in a centrifuged system to bone matrix promotion by osteoconductivity and osteoinductivity (as seen in hBMSC conversion into cells that secrete bone-specific matrix) in parallel.

Therefore, vaterite microsponges via the unique feature of integrating functional biological molecules into their crystalline structure are a potential packing material in translational medicine, and clinical applications such as in bone defects 
with potent osteoinductivity accelerate the bone growth and regeneration.

\section{Conflicts of interest}

There are no conflicts to declare.

\section{Acknowledgements}

This study was undertaken in the laboratory established and run by Prof. Richard O. C. Oreffo, at the Centre for Human Development, Stem Cells and Regeneration, University of Southampton, UK. D. W. G. wishes to thank Prof. Oreffo for his lab support and encouragement during the study. In addition, D. W. G. wishes to thank Prof. Stephen Mann for providing the groundwork for the study and providing plenty of inspiration in biomimetic and bioinspired materials chemistry.

\section{Notes and references}

1 A. S. Brydone, D. Meek and S. Maclaine, Proc. Inst. Mech. Eng., Part H, 2010, 224, 1329.

2 V. Campana, G. Milano, E. Pagano, M. Barba, C. Cicione, G. Salonna, W. Lattanzi and G. Logroscino, J. Mater. Sci.: Mater. Med., 2014, 25, 2445.

3 F. Jordana, C. Le Visage and P. Weiss, Med. Sci., 2017, 33, 60.
4 C. A. Rossi, M. Pozzobon and P. De Coppi, Organogenesis, 2010, 6, 167.

5 D. W. Green, Biomaterials, 2008, 30(10), 1918.

6 E. Schepers, M. de Clercq, P. Ducheyne and R. Kempeneers, J. Oral Rehabil., 1991, 18, 439.

7 J. Chou, et al., Adv. Eng. Mater., 2011, 12, 1.

8 J. Henkel, M. A. Woodruff and D. R. Epari, et al., Bone Res., 2013, 1, 216.

9 R. Lomas, A. Chandrasekar and T. N. Board, Hip Int., 2013, 23, 427.

10 D. W. Green, et al., Adv. Funct. Mater., 2005, 15(6), 917.

11 E. Weber and B. Pokroy, CrystEngComm, 2015, 17, 5873.

12 N. Watabe, J. Ultrastruct. Res., 1965, $12,351$.

13 D. V. Oliveira, T. S. Silva and O. D. Cordeiro, et al., Sci. World J., 2012, 765909.

14 C. Milet, S. Berland, M. Lamghari and L. Mouries, et al., C. R. Palevol, 2004, 493.

15 D. Walsh, B. Lebeau and S. Mann, Adv. Mater., 1999, 11, 324. 16 D. W. Green, D. Walsh, X. B. Yang, S. Mann and R. O. C. Oreffo, J. Mater. Chem., 2004, 14, 2206-2213.

17 D. W. Green, J. Biomed. Mater. Res., Part A, 2014, 103, 2885.

18 M. J. Almeida, C. Milet and J. Peduzzi, et al., J. Exp. Zool., 2000, 288, 327.

19 M. Rousseau, L. Pereira-Mouriès, M. J. Almeida, C. Milet and E. Lopez, Comp. Biochem. Physiol., Part B: Biochem. Mol. Biol., 2003, 135, 1. 